\title{
UKIRT CGS3 OBSERVATIONS OF NEW IRAS 21 MICRON SOURCES
}

\author{
SUN KWOK \\ Dept. of Physics 83 Astronomy, University of Calgary, Calgary, Canada \\ BRUCE J. HRIVNAK \\ Dept. of Physics 85 Astronomy, Valparaiso Univerity, Indiana, U.S.A. \\ TOM R. GEBALLE \\ Joint Astronomy Center, Hilo, Hawaii, U.S.A. \\ and \\ PHILLIP L. LANGILL \\ Dept. of Physics 83 Astronomy, University of Calgary, Calgary, Canada
}

An unidentified emission feature at $21 \mu \mathrm{m}$ has been detected in the IRAS Low Resolution Spectra (LRS) of 5 IRAS sources (Kwok, Volk, and Hrivnak 1989, Hrivnak and Kwok 1991). The sources are generally found to be F and $G$ supergiants with cool, detached dust shells. We have searched for additional $21 \mu \mathrm{m}$ sources in the LRS database and have obtained ground-based UKIRT spectra at 10 and $20 \mu \mathrm{m}$ in an attempt to confirm the LRS feature.

The LRS spectra of $05113+1347,20000+3239$, and $22223+4327$ show a peak around $21 \mu \mathrm{m}$, a flat plateau between 12 and $18 \mu \mathrm{m}$, and a drop between 7 and $11 \mu \mathrm{m}$ which are the characteristics of $21 \mu \mathrm{m}$ sources such as $07134+1005$. UKIRT CGS3 spectra of these 3 sources plus $05341+0852$ have been obtained with spectral resolution of CGS3 is $\sim 52$ at $10 \mu \mathrm{m}$ and $\sim 72$ at 20 $\mu \mathrm{m}$. The spectra of the $05341+0852$ is different from the others in that the $10 \mu \mathrm{m}$ band is higher than the $20 \mu \mathrm{m}$ band. We are therefore less certain about the $21 \mu \mathrm{m}$ feature in this object.

Ground-based visible, near- and mid-infrared photometry have been obtained for the $21 \mu \mathrm{m}$ sources. The energy distribution of the sources show the "double-peaked" distribution characteristic of proto-planetary nebulae (see Kwok, this volume).

All the $21 \mu \mathrm{m}$ sources have been found to show carbon-rich photosphere with $\mathrm{C}_{2}$ and/or $\mathrm{C}_{3}$ features (see Hrivnak IV:120). Unidentified 3.4-3.5 $\mu \mathrm{m}$ features are also observed in addition to the $3.3 \mu \mathrm{m}$ PAH feature (Geballe et al. 1992). The strength of the $21 \mu \mathrm{m}$ feature implies that it originates from an abundant element. The carbon-rich nature of the sources suggests that the carbon atom may be a major constituent of the molecule/grain responsible for the $21 \mu \mathrm{m}$ feature.

\section{References}

Geballe, T.R., Tielens, A.G.G.M., Kwok, S., \& Hrivnak, B.J. 1992, ApJ, 387, L89

Hrivnak, B.J., \& Kwok, S. 1991, ApJ, 368, 564

Kwok, S., Volk, K., Hrivnak, B.J. 1989, ApJ, 345, L51 